\title{
In vivo Time domain Broadband (600 -1200 nm) Diffuse Optical Characterization of Human Bone
}

\author{
Sanathana Konugolu Venkata Sekar ${ }^{1}$, Alberto Dalla Mora ${ }^{1}$, Edoardo Martinenghi ${ }^{1}$, , Paola Taroni ${ }^{1}$, Antonio \\ Pifferi $^{1}$, Andrea Farina ${ }^{1,2}$, Jordi Puig ${ }^{3}$, Eugènia Negredo ${ }^{3,4}$, Claus Lindner ${ }^{5}$, Marco Pagliazzi ${ }^{5}$, Turgut \\ Durduran $^{5}$ \\ ${ }^{1}$ Politecnico di Milano, Dipartimento di Fisica, Milano, Italy \\ ${ }^{2}$ Consiglio Nazionale delle Ricerche - Istituto di Fotonica e Nanotecnologie,Milano \\ ${ }^{3}$ Unitat VIH, Fundació Lluita contra la SIDA, Hospital Germans Trias i Pujol, Universitat Autònoma de Barcelona, Badalona, Barcelona, Spain \\ ${ }^{4}$ Universitat de Vic-Universitat Central de Catalunya \\ ${ }^{5}$ ICFO-Institut de Ciències Fotòniques, The Barcelona Institute of Science and Technology, 08860 Castelldefels (Barcelona), Spain \\ sanathana.konugolu@polimi.it
}

\begin{abstract}
We present an in-vivo characterization performed on 53 subjects in 6 different superficial bone tissue locations of human body using a broadband time-resolved diffuse optical system, designed specifically for real-time clinical measurements.

(C) 2015 Optical Society of America

OCIS codes: $170.5280 ; 170.6510 ; 170.6935$.
\end{abstract}

\section{Introduction}

In recent decades, with ageing population, the science has found an increase in demand for bone related diagnosis and treatment tools. Noninvasive nature and high penetration depth of diffuse optical methods were applied successfully to various domains of life sciences: optical mammography [1], brain oximetry [2], in vivo tissue characterization [3] are just few instances. Though there are few optical studies of bone tissues, they lack either the broadband nature [4] or number of subjects [5]. The gold standard for bone mineral assessment Dual X-ray Absorptiometry (DEXA) [6], cautions its radiation invasiveness by using ionizing $\mathrm{x}$-rays. Few studies even question the relevance of bone mineral density to bone related pathologies. Ultrasound imaging [7], provides good morphological information, but lags in judging the physiological condition of bone. Bone mineral assessment using MRI has been reported [8], but high cost and non-portable bulk nature prevents its successful widespread implementation.

Diffuse optical methods have proved to quantify collagen, the dominant connecting tissue in human bones. DEXA scans are applied on bones of high porosity (50-90\%) called trabecular bones, wherein bone pathological changes are reveled earlier. Few of the trabecular bones namely, radius distal (RD), radius proximal (RP), ulna distal (UD), ulna proximal (UP), trochanter (T), calcaneus (C) belong to the DEXA compatible spongy bone category. Interestingly, the superficial nature of these bones $(7-15 \mathrm{~mm}$ depth below the skin) makes them ideal candidates for diffuse optical studies.

In this work, we present the hemodynamics (oxy, deoxy-hemoglobin, oxygen saturation) and tissue constituents (lipid, water, collagen) assessment on the above mentioned 6 bony prominence locations. Except calcaneus all other measurements were performed in reflectance geometry with $25 \mathrm{~mm}$ as source detector separation to allow maximum penetration depth into bone tissues.

\section{Methods and Material}

\subsection{Instrumentation and Data Analysis}

The Instrumentation consists of supercontinuum fiber laser (SC450, Fianium, UK) which empowers the system with the broadband source (450-1750 nm). Light tunability is achieved by a Pellin Broca prism, which disperses the light into the source fiber. Two detectors, a SiPM (Silicon Photomultiplier, Hamamatsu, S10362-11-050C with homemade front-end electronics) and an InGaAs PMT (Photomultiplier Tube, Hamamatsu, mod.H10330A-45), equips the system with very high flat broadband $(600-1350 \mathrm{~nm})$ responsivity. For this study we limited our measurement range to $600-1200 \mathrm{~nm}$, owing to strong water absorption that strongly affect the signal level above $1200 \mathrm{~nm}$. A drift and distortion control strategy enabled the real time operation of the system in clinical environment. More details on system construction and validation can be found in Ref.[9]. 

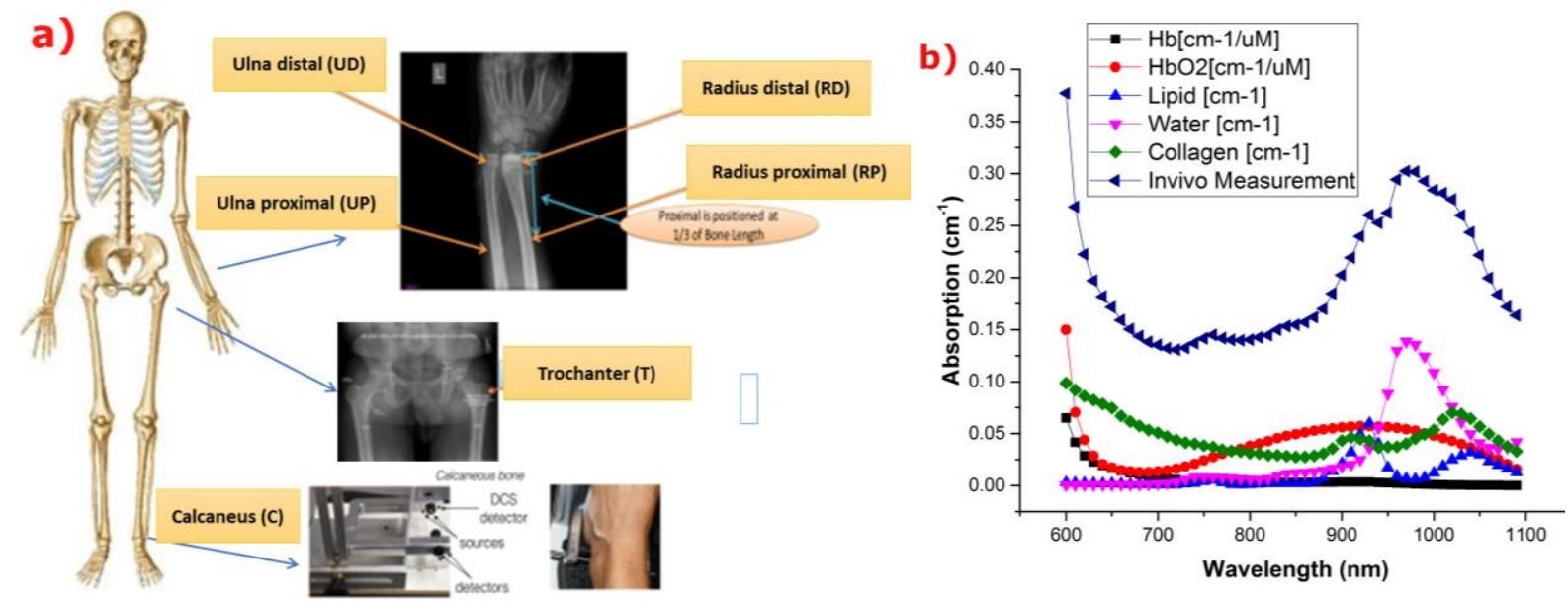

Fig. 1.(a) Diagrammatic view of measured tissue locations, (b) Secondary fit depicting the contribution of each tissue constituent to an arbitrary in vivo spectrum.

Absorption $\left(\mu_{\mathrm{a}}\right)$ and reduced scattering coefficients $\left(\mu \mathrm{s}^{\prime}\right)$ were obtained by fitting acquired curves to an analytical model of the Diffusion Equation [10]. Homogeneous semi-infinite medium and finite slab thickness models were used for reflectance and transmittance geometries, respectively. For better accuracy, extrapolated boundary conditions were used. Depth penetrated photons were taken into account by fixing the fit range from $80 \%$ of the rising edge to $1 \%$ of the falling edge. More details on the fitting model are described in Ref: [11]. The scattering spectrum was approximated to the empirical power law derived from Mie theory [12]: $\mu_{\mathrm{s}}{ }^{\mathrm{a}}=\mathrm{a}\left(\lambda / \lambda_{0}\right)^{-\mathrm{b}}$, in which $\mathrm{a}$ and $\mathrm{b}$ characterize scatterer density and size respectively, with $\lambda_{0}=600 \mathrm{~nm}$, the fitting was performed in the range of 600 $850 \mathrm{~nm}$. Key tissue constituents were quantified by fitting the in vivo absorption spectrum with the linear combination spectra of major tissue constituents. Fig. 1(b) shows the weighted tissues constituent (Lipid, water, collage, oxy-, deoxy-hemoglobin) spectra corresponding to an in vivo spectrum measurement.

\subsection{Measurement Protocol}

Fig 1(a) shows the in-vivo bone locations measured by the protocol. 53 subjects spanning the age of 20-78 years were recruited for this study. The protocol and instrumentation was approved by the ethical committee (Comite de Ética de la Investigación del Hospital Germans Trias i Pujol, Badalona, Spain). Along with the health questionnaire recording the health history of the subject, an informed consent was duly signed before the measurement on the subjects. To track the general health status during the protocol period, blood pressure and pulse rate were monitored before and after the measurement. Measurement on calcaneus (C) was performed with specially designed custom probe in transmittance geometry. Reflectance geometry with $25 \mathrm{~mm}$ as source detector separation was used for RD, RP, UD, UP and T locations.

\section{Results and Discussion}

The average broadband absorption spectra of measured locations are shown in Fig 2(a). For readability, only 4 out of 6 locations are shown (radius proximal, and ulna distal are not shown).
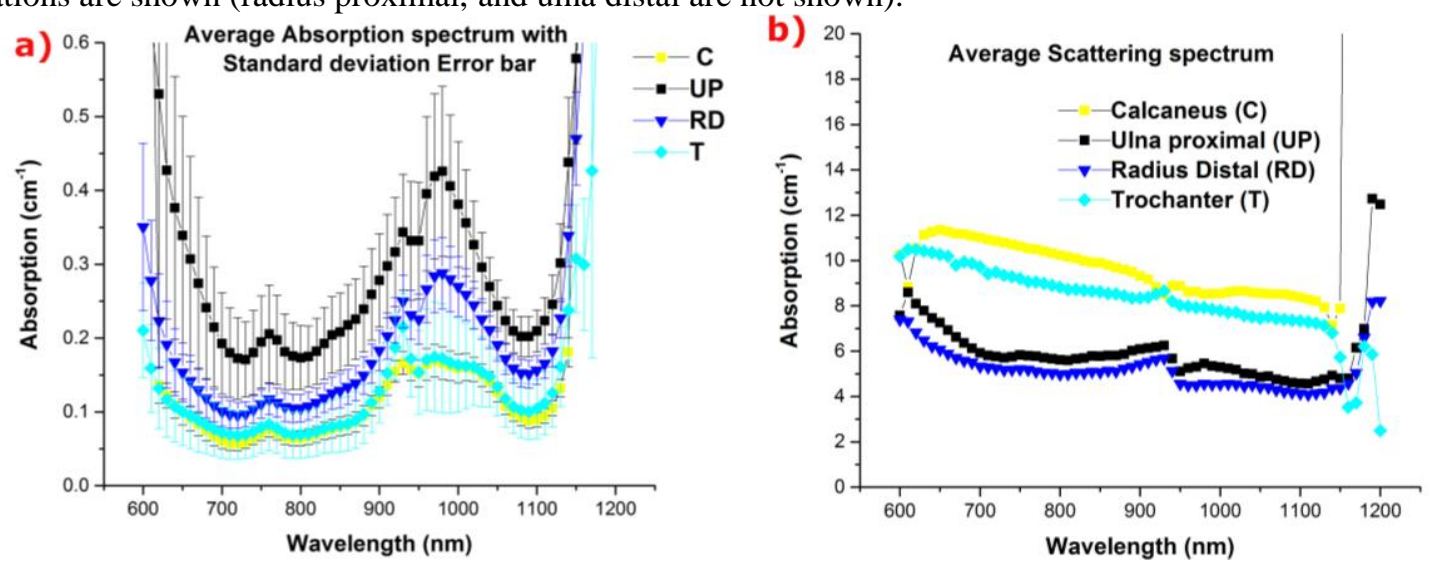

Fig. 2.(a) Average absorption spectrum with standard deviation of inter subject variation as error bars, (b) Average $\mu_{\mathrm{s}}$ ' spectrum of 4 in vivo bone locations: calcaneus, ulna proximal, radius distal, and trochanter. 
Inter-subject variability is seen high for trochanter region, contrary to calcaneus with low variation. Proximal locations seem to have comparatively a high average absorption compared to the distal locations. Overall trochanter and calcaneus possess a low average absorption compared to other locations, whereas a high value is seen in their average $\mu \mathrm{s}^{\prime}$ spectrum Fig 2(b). A slight variation is seen among the average scattering spectra of the distal and proximal locations, with least value shown by the radius distal location.

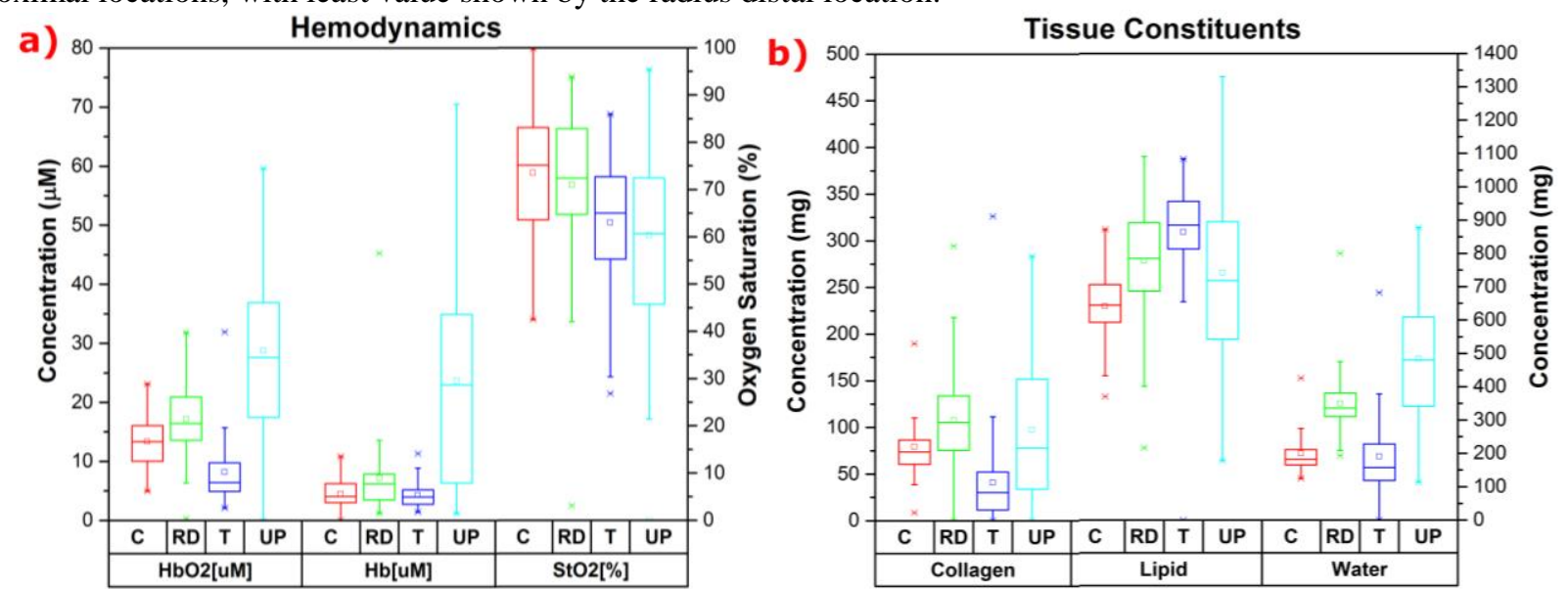

Fig. 3 (a) Box plot comparison of hemodynamics parameter, left y-axis corresponds to $\mathrm{HbO} 2$ and $\mathrm{Hb}$ values while the right to $\mathrm{StO} 2$ (\%). (b) Box plot depiction of tissue constituents, left y-axis corresponds to collagen and right to lipid-water concentration in $\mathrm{mg}$.

From Fig. 3, proximal locations show a high blood volume compared to distal locations. Least blood volume and high lipid content reveals the adipose nature of trochanter tissue. High collagen content coupled with relatively less lipid content of proximal locations suggest the possibility of choosing it for future bone related pathological studies. In general, Oxygen saturation lies between 60-80\%. In particular, ulna proximal shows the least average value and high inter-subject variability.

Work is in progress to correlate optically derived information with bone tissue physiology.

\section{Acknowledgements}

The research leading to these results has partially received funding from the EC's Seventh Framework Programme under the projects OILTEBIA (grant agreement n. 317526), Fundació CELLEX Barcelona, Obra social "la Caixa" Foundation (LlumMedBcn), "Severo Ochoa" (SEV-2015-0522).

\section{References}

[1] P. Taroni, A. Pifferi, E. Salvagnini, L. Spinelli, A. Torricelli, and R. Cubeddu, "Seven-wavelength time-resolved optical mammography extending beyond $1000 \mathrm{~nm}$ for breast collagen quantification," Opt. Express, vol. 17, no. 18, pp. 15932-15946, 2009.

[2] A. Torricelli, D. Contini, A. Pifferi, M. Caffini, R. Re, L. Zucchelli, and L. Spinelli, "Time domain functional NIRS imaging for human brain mapping," Neuroimage, vol. 85, pp. 28-50, 2014.

[3] R. M. Doornbos, R. Lang, M. C. Aalders, F. W. Cross, and H. J. Sterenborg, "The determination of in vivo human tissue optical properties and absolute chromophore concentrations using spatially resolved steady-state diffuse reflectance spectroscopy.," Phys. Med. Biol., vol. 44, pp. 967-981, 1999.

[4] P. Farzam, C. Lindner, U. M. Weigel, M. Suarez, A. Urbano-Ispizua, and T. Durduran, "Noninvasive characterization of the healthy human manubrium using diffuse optical spectroscopies.," Physiol. Meas., vol. 35, no. 7, pp. 1469-91, 2014.

[5] A. Pifferi, A. Torricelli, P. Taroni, A. Bassi, E. Chikoidze, E. Giambattistelli, and R. Cubeddu, "Optical biopsy of bone tissue: a step toward the diagnosis of bone pathologies.," J. Biomed. Opt., vol. 9, no. 3, pp. 474-80, 2004.

[6] Y. Lu, H. K. Genant, J. Shepherd, S. Zhao, a Mathur, T. P. Fuerst, and S. R. Cummings, "Classification of osteoporosis based on bone mineral densities.," J. Bone Miner. Res., vol. 16, no. 5, pp. 901-10, 2001.

[7] S. H. Prins, H. L. Jørgensen, L. V Jørgensen, and C. Hassager, "The role of quantitative ultrasound in the assessment of bone: a review.," Clin. Physiol., vol. 18, no. 1, pp. 3-17, 1998.

[8] T. M. Link, S. Majumdar, P. Augat, J. C. Lin, D. Newitt, Y. Lu, N. E. Lane, and H. K. Genant, "In vivo high resolution MRI of the calcaneus: differences in trabecular structure in osteoporosis patients.," J. Bone Miner. Res., vol. 13, no. 7, pp. 1175-82, 1998.

[9] S. Konugolu Venkata Sekar, A. Farina, E. Martinenghi, A. Dalla Mora, P. Taroni, A. Pifferi, T. Durduran, M. Pagliazzi, C. Lindner, P. Farzam, M. Mora, M. Squarcia, and A. Urbano-Ispizua, "Broadband time-resolved diffuse optical spectrometer for clinical diagnostics: characterization and in-vivo measurements in the 600-1350 nm spectral range," vol. 9538, p. 95380R, 2015.

[10] M. S. Patterson, B. Chance, and B. C. Wilson, "Time resolved reflectance and transmittance for the non- invasive measurement of tissue optical properties," Appl. Opt., vol. 28, no. 12, pp. 2331-2336, 1989.

[11] D. Contini, F. Martelli, and G. Zaccanti, "Photon migration through a turbid slab described by a model based on diffusion approximation. I. Theory,” Appl. Opt., vol. 36, no. 19, pp. 4587-4599, 1997.

[12] J. R. Mourant, T. Fuselier, J. Boyer, T. M. Johnson, and I. J. Bigio, "Predictions and measurements of scattering and absorption over broad wavelength ranges in tissue phantoms.," Appl. Opt., vol. 36, no. 4, pp. 949-57, 1997. 